\title{
Impacto de la evaluación infectológica en el uso racional de antimicrobianos en pacientes con bacteriemia por Streptococcus pneumoniae
}

\author{
Sergio Mella M., Rodrigo Blamey D., Oriana Paiva M., Jorge Yáñez V., Gisela Riedel M., \\ Mabel Aylwin R., Hanriete Chabouty G. y Carlos Ortega R.
}

\section{Universidad de Concepción,}

Facultad de

Departamento de Medicina

Interna (SMM, RBD, GRM, MAR)

Hospital Clínico Regional

de Concepción, Chile

Servicio Medicina

Interna (OPM, JYV)

Unidad de Infectología (SMM, RBD)

Laboratorio de

Microbiología (HCHG)

Unidad de Paciente Crítico (COR)

Financiamiento: Proyecto Dirección de Investigación, Universidad de

Concepción, DIUC 205.085.029-1.0

Recibido: 15 de junio de 2006

Aceptado: 24 de abril de 2007

Correspondencia a: Sergio Mella Montecinos pignatio@vtr.net

\section{Impact of infectious diseases specialist evaluation on the rational use of antibiotics in patients with Streptococcus pneumoniae bacteremia}

The increasing reporting of Streptococcus pneumoniae resistance to penicillin has lead to the recommendation of third generation cephalosporins for the treatment of serious pneumococcal infections. As a consequence, clinicians usually do not prescribe first line antibiotics despite the availability of susceptibility studies. With the aim to evaluate the impact of the infectious diseases specialist evaluation in the appropriate use of the susceptibility studies, two series were compared: a historic control one $(1998-2002, \mathrm{n}=50)$ and a prospective group that had the advisement of one infectious diseases specialist (2003-2006, $n=60)$. In both series, pneumonia stood out as the source of the bacteremia, and alcoholism/hepatic insufficiency as associated factor. In the first series, 48 isolates resulted susceptible to penicillin by agar diffusion, and 1 out of 36 patients with pneumonia had a change in the antibiotic therapy to penicillin. In the prospective series, we found 56 susceptible isolates, and the remaining four were classified as intermediate susceptibility according to the MIC value. Antibiotics were changed in 36 out of 50 patients with bacteremic pneumococcal pneumonia $(\mathrm{p}<0.05)$. The infectious diseases specialist evaluation had a positive impact on the use of the microbiological information for therapeutics purposes.

Key words: Pneumococcal pneumonia, bacteremia, penicillin, resistance.

Palabras claves: Neumonía neumocóccica, bacteriemia, penicilina, resistencia.

\section{Introducción}

$\mathbf{L}$ a infección invasora producida por Streptococcus pneumoniae se asocia todavía a una elevada morbilidad y mortalidad ${ }^{1}$. De particular importancia es el compromiso del torrente circulatorio producido por este patógeno ${ }^{2-4}$, que en el caso de las neumonías, se asocia a un pronóstico ominoso ${ }^{5,6}$. A pesar del progreso médico, la mortalidad de la neumonía neumocóccica bacteriémica en adultos varía en la literatura médica entre 6 y $40 \% \%^{4,7-9}$. Por otra parte, el aumento de resistencia de $S$. pneumoniae frente a antimicrobianos de primera línea, como penicilinas y macrólidos, ha derivado en la recomendación del uso de cefalosporinas de tercera generación para el tratamiento de infecciones graves producidas por este microorganismo ${ }^{10-12}$.

En nuestro medio, en los estudios de infecciones neumocóccicas invasoras realizados en la población pediátrica, se determinaron los serotipos capsulares de mayor importancia y se describió una proporción importante de aislados con resistencia a penicilina ${ }^{13,14}$; mientras que, en las series publicadas en adultos, hay escasa información sobre serotipificación y la resistencia a penicilina es menos frecuente ${ }^{15-20}$. Más aún, hay antecedentes de una clara dicotomía, al comparar la población pediátrica con la adulta, en la susceptibilidad de aislados de $S$. pneumoniae frente a $\beta$-lactámicos ${ }^{21}$. A pesar de lo anterior, en Chile esta realidad epidemiológica concreta no ha sido necesariamente explicitada en las recomendaciones para el tratamiento de la neumonía adquirida en la comunidad ${ }^{22,23}$.

Por otra parte, hay todavía una fuerte reticencia en el médico clínico a utilizar la información microbiológica, cuando ella está disponible, en el tratamiento de una serie de infecciones, siendo uno de los ejemplos más interesantes, la infección neumocóccica ${ }^{24-26}$. Se ha planteado también que, dada la escasa utilidad clínica de los hemocultivos, este tipo de estudio microbiológico no sería necesario en el manejo de la neumonía neumocóccica $^{25}$

Dado el contexto actual de resistencia y la falta de desarrollo de nuevos antibacterianos, es particularmente importante, a nivel hospitalario, el control del 
uso de antimicrobianos por un equipo especializa$\mathrm{do}^{27,28}$.

Sobre la base de lo anterior, comparamos en nuestro hospital, dos períodos: 1998-2002 y 2003-2006, respecto del impacto de la evaluación practicada por el médico infectólogo en el uso racional de antimicrobianos en la infección neumocócica bactierémica.

\section{Pacientes y Método}

Se realizó un estudio comparativo, secuencial, entre un control histórico y una serie prospectiva de pacientes hospitalizados en el Servicio de Medicina Interna o Unidad de Cuidados Intensivos Médicos del Hospital Clínico Regional de Concepción (HCRC) -hospital universitario de nivel terciario y centro de derivación de la Octava Región del país, con aproximadamente mil camas- que cursaron con bacteriemia por $S$. pneumoniae en dos períodos: años 1998 a 2002 y octubre 2003 a abril 2006, con el fin de evaluar el uso racional de antimicrobianos, de acuerdo con la información microbiológica disponible.

El primer grupo de pacientes (1998-2002) se consideró como control histórico, dado que no hubo una evaluación infectológica sistemática de estos enfermos y fueron estudiados en forma retrospectiva sobre la base de revisión de fichas clínicas. Nuestro hospital no contaba, en ese entonces, con horas asistenciales de Infectología (sólo con 22 horas universitarias a la semana desde junio de 2002).

El segundo grupo, serie prospectiva, fue evaluado desde octubre de 2003, coincidiendo con la formación de la Unidad de Infectología en nuestro centro -dado el reconocimiento de la importancia asistencial de esta sub-especialidad- se contó con un total de 55 horas semanales asistenciales, repartidas entre los dos autores principales de este estudio (SMM 11 horas y RBD 44 horas).

Todos los pacientes con bacteriemia neumocóccica, en este período, fueron evaluados formal y sistemáticamente por un infectólogo y se les realizó seguimiento clínico con control ambulatorio al alta. Esta evaluación incluyó el énfasis en el uso de la información microbiológica disponible; en general, en todos los pacientes con infecciones extra-meníngeas, se intentó la suspensión del uso de ceftriaxona -en nuestro centro todos los pacientes con neumonía que se hospitalizan, inician tratamiento con esta cefalosporina- y uso de bencilpenicilina $G$ sódica o, cuando la condición clínica del paciente lo permitiera, uso de amoxicilina oral.

Se consideró bacteriemia neumocóccica el aislado, en una o dos muestras de hemocultivos, de cocos grampositivos, lanceolados, agrupados en pares o cadenas cortas, catalasa negativa, susceptibles a optoquina y con pruebas de solubilidad en bilis positiva ${ }^{29}$. En este protocolo no se incluyeron cepas con propiedades fenotípicas de difícil interpretación ${ }^{30}$.

Se estudiaron también aquellas co-morbilidades asociadas a bacteriemia neumocóccica validadas por la literatura médica: enfermedad pulmonar obstructiva crónica y secuela post TBC, alcoholismo y daño hepático crónico, diabetes mellitus, infección por VIH/SIDA, esplenectomía, neoplasia, tratamiento inmusupresor y tabaquismo $^{31-33}$. Se estudió la susceptibilidad de los aislados de $S$. pneumoniae por difusión en $\operatorname{agar}^{34}$; en la mayoría de las cepas procedentes de la serie prospectiva se determinó la CIM mediante E-test. Se clasificó la susceptibilidad de acuerdo con los criterios del Clinical Laboratory Standards Institute, formalmente NCCLS ${ }^{35}$.

Mortalidad cruda fue definida como el fallecimiento del paciente con infección del torrente circulatorio por $S$. pneumoniae, durante la hospitalización, sin que necesariamente fuera consecuencia de la infección neumocóccica.

Se usó $\chi^{2}$ para comparar el uso de penicilinas, de acuerdo con el estudio de susceptibilidad, entre la serie histórica y la prospectiva.

\section{Resultados}

Control histórico. La revisión de los libros de hemocultivos correspondientes al período 1998-2002, determinó 72 episodios de bacteriemia neumocóccica. De este número, se pudieron recuperar los datos clínicos de 50 pacientes con hemocultivos positivos para S. pneumoniae. Predominó el sexo masculino (72\%) y el grupo etario entre 31 y 60 años $(60 \%)$; los pacientes sobre 60 años representaron el 34\%. De las co-morbilidades destacó el daño hepático crónico y/o alcoholismo (56\%), enfermedad pulmonar obstructiva crónica, secuela post TBC o tabaquismo (32\%), inmunosupresión y/o cáncer (26\%) y diabetes mellitus (16\%) (Tabla 1). El cuadro que originó la bacteriemia fue neumonía en $72 \%$ de los casos; en $8 \%$ MBA y en $14 \%$ no se logró determinar el foco infeccioso asociado a la bacteriemia.

Se encontraron 48 cepas de $S$. pneumoniae susceptibles a penicilina y resistencia, por difusión en agar, en dos aislados. En un paciente que cursó con neumonía y cuyo aislado era susceptible a penicilina, se modificó la terapia antimicrobiana con suspensión de ceftriaxona y uso de penicilina sódica, después de evaluación por uno de los autores (SMM). La mortalidad cruda fue de $24 \%$ (12 casos). 
Serie prospectiva. Se incluyeron 60 pacientes; $65 \%$ eran de sexo masculino. También predominó el grupo etario entre 31 y 60 años (47\%); los pacientes sobre 60 años representaron el $43 \%$ de esta cohorte. Tal como se señala en la Tabla 1, el estudio de las co-morbilidades asociadas, nuevamente, demostró la importancia del

Tabla 1. Comparación de comorbilidades entre serie histórica y prospectiva

\begin{tabular}{lcc|} 
& $\begin{array}{c}\text { Control histórico } \\
(\mathbf{n}=\mathbf{5 0})\end{array}$ & $\begin{array}{c}\text { Serie prospectiva } \\
(\mathbf{n}=\mathbf{6 0})\end{array}$ \\
Comorbilidad & $\mathbf{\%}$ & $\mathbf{\%}$ \\
Alcoholismo-daño hepático crónico & 56 & 45 \\
EPOC-tabaquismo-secuela TBC & 32 & 37 \\
Inmunosupresión-cáncer & 26 & 13 \\
Diabetes mellitus & 16 & 10 \\
\hline EPOC: enfermedad pulmonar obstructiva crónica & \\
\hline
\end{tabular}

Tabla 2. Variables microbiológicas de los aislados de Streptococcus pneumoniae, serie prospectiva $(n=60)$

\begin{tabular}{|c|c|c|}
\hline & & CIM $\mu \mathrm{g} / \mathrm{ml}$ \\
\hline Total de aislados resistentes por difusión en agar & $4 / 60$ & \\
\hline Aislados resistentes por difusión en agar-foco MBA & $2 / 60$ & $(0,125,0,125)$ \\
\hline Aislados resistentes por difusión en agar-foco neumonía & $2 / 60$ & $(0,25,0,75)^{*}$ \\
\hline Aislados susceptibles por difusión en agar-foco MBA & $4 / 60$ & $(0,004,0,02,0,012,0,018)$ \\
\hline Aislados susceptibles por difusión en agar-foco neumonía & $48 / 60$ & $(0,0012-0,06)$ \\
\hline \multicolumn{3}{|c|}{$\begin{array}{l}\text { MBA= meningitis bacteriana aguda } \\
\text { *Según el Drug-resistant Streptococcus pneumoniae Therapeutic Group estos dos aislados serían } \\
\text { susceptibles a penicilina (52) }\end{array}$} \\
\hline
\end{tabular}

Tabla 3. Número de pacientes, de serie prospectiva, con neumonía a los cuales se les suspendió cefalosporina de tercera generación y se indicó bencilpenicilina $\mathrm{G} \circ$ amoxicilina

\begin{tabular}{|c|c|c|}
\hline $\begin{array}{l}\text { Terapia inicial } \\
\text { Ceftriaxona } \\
\text { (1-2 gr al día IV)* } \\
\qquad n\end{array}$ & $\begin{array}{l}\text { Modificación de terapia con } \\
\text { Penicilina G } \\
(3.000 .000 \text { UI c/6 h IV)* } \\
n\end{array}$ & $\begin{array}{l}\text { rmación microbiológica } \\
\text { Amoxicilina } \\
(1 \text { gramo } x 3 \text { v PO)* } \\
n\end{array}$ \\
\hline 50 & $\begin{array}{l}27 \\
2 \text { ** }\end{array}$ & 9 \\
\hline
\end{tabular}

Causas de no modificación de terapia

ALPS 5

Insuficiencia renal aguda 3

Sepsis fulminante 1

Condición crítica

Resistencia***

5
3
1
1
2

* Dosis estándar. ** En 2 pacientes se suspendió ceftriaxona y se indicó penicilina G, pero ésta debió discontinuarse por reacción alérgica. *** Difusión en agar.

ALPS: alcoholismo, leucopenia, sepsis neumocóccica alcoholismo y/o daño hepático crónico presentes en $45 \%$ de los enfermos; tabaquismo, EPOC y/o secuela post $\mathrm{TBC}$ con $37 \%$; inmunosupresión y/o cáncer en $13 \%$ y diabetes mellitus presente en $10 \%$ de esta cohorte. El cuadro clínico más frecuentemente identificado como origen de la bacteriemia, fue neumonía en $83 \%$ y en segundo lugar MBA con una frecuencia de $10 \%$.

De los aislados, 56 fueron susceptibles a penicilina por difusión en agar; con respecto a las cuatro cepas resistentes por esta técnica, el estudio por E-test demostró CIMs de 0,125- 0,125- 0,25 y 0,75 $\mu \mathrm{g} / \mathrm{ml}$, respectivamente (susceptibilidad intermedia de acuerdo al CLSI).

Es interesante señalar que, en dos casos de MBA, dos aislados fueron informados como resistentes a penicilina por difusión en agar; sin embargo, el estudio por E-test demostró susceptibilidad intermedia $(0,125$ $\mu \mathrm{g} / \mathrm{ml})$. El resto de los cuatro aislados de foco meníngeo fueron susceptibles a penicilina (Tabla 2). La mortalidad cruda en esta segunda serie fue de $25 \%$ (15 pacientes).

En la serie estudiada prospectivamente, de 50 pacientes con neumonía neumocóccica bacteriémica, se suspendió ceftriaxona y se indicó penicilina $G$ sódica en 27 pacientes y en 9 se suspendió la cefalosporina de tercera generación y se indicó amoxicilina (Tabla 3). La causa más frecuente de no suspender ceftriaxona e indicar una penicilina fue el síndrome de alcoholismo, leucopenia, sepsis neumocóccica (ALPS) ${ }^{36}$ en cinco pacientes.

Otros motivos de no cambio de terapia fueron: insuficiencia renal aguda, por la posibilidad de neurotoxicidad con dosis elevadas de penicilina, en tres pacientes; un paciente falleció en forma fulminante (no cumpliendo criterios de ALPS) y otro por su condición crítica, se mantuvo en tratamiento con ceftriaxona, falleciendo al cuarto día de hospitalización; y dos pacientes por presencia de aislados resistentes por difusión en agar (susceptibilidad intermedia por E-test). Además, en dos pacientes se suspendió la ceftriaxona, pero el uso de penicilina produjo una reacción alérgica, debiéndose indicar, finalmente, un antibiótico no $\beta$ lactámico.

\section{Discusión}

Nuestro trabajo tiene como limitación mayor el diseño elegido, al realizar un estudio comparativo, secuencial, entre una serie prospectiva y un control histórico. De hecho, no se pudieron recuperar los datos de todas las bacteriemias documentadas microbiológicamente en la serie histórica; lo que debe haber influido en la 
escasa descripción de síndrome de ALPS y en diferencias demográficas menores. Además, en nuestros resultados se estudió la mortalidad que se producía durante toda la estancia hospitalaria que no necesariamente fue atribuible a la infección neumocóccica, propiamente tal. Sin embargo, el objetivo principal del estudio, cuantificar el uso de la información microbiológica en el tratamiento de la infección neumocóccica bacteriémica, claramente se cumplió y nuestros resultados confirman la utilidad de la evaluación infectológica, en privilegiar el uso de penicilinas en vez de ceftriaxona.

Así, basta comparar que en el control histórico a sólo un enfermo con neumonía se le suspendió la ceftriaxona y se indicó penicilina -justamente evaluado por uno de los autores- mientras que en la serie prospectiva en 36 de 50 pacientes se usaron penicilinas; incluso hubo dos enfermos más a los que se le suspendió la ceftriaxona y se indicó penicilina $G$, la que tuvo que suspenderse por reacción alérgica.

La idea fuerza de disminuir el uso de cefalosporinas y privilegiar el uso de antimicrobianos que generen menor presión selectiva, es uno de los objetivos más relevantes de la unidad de Infectología en nuestro hospital; así, enfatizamos el uso de la información microbiológica para el tratamiento de infecciones como peritonitis secundaria, pielonefritis aguda e infección de piel y tejidos blandos. De hecho, en el HCRC se normó el uso de gentamicina más metronidazol como esquema de tratamiento antimicrobiano para cuadros de peritonitis secundaria ${ }^{37,38}$; en nuestra normativa se contraindica el uso de aminoglucósidos-aminociclitoles en pacientes sobre 65 años de edad, que estén evolucionando con shock séptico y/o fracaso renal. En pielonefritis aguda, el fundamento es similar, debiéndose considerar la descripción creciente de resistencia a quinolonas y cefalosporinas de tercera generación en aislados procedentes de la comunidad ${ }^{39}$; las contraindicaciones para el uso de aminoglucósidos son similares a las descritas para peritonitis secundaria. Finalmente, en infección de piel y tejidos blandos compartimos el punto de vista señalado por $W_{\text {olff }}{ }^{40}$ sobre la utilidad de la combinación de penicilina más cloxacilina para el tratamiento de estas infecciones en pacientes inmunocompetentes y que no tienen elementos clínicos sugerentes de infección necrosante.

Desde otra perspectiva, destaca en este estudio la importancia que adquiere, en la Región del Bío-Bío, el alcoholismo y el daño hepático crónico como comorbilidad asociada a bacteriemia neumocóccica, en comparación con series estudiadas en la Región Metropolitana ${ }^{16-18}$, pero que resulta similar a lo descrito en la zona sur de Chile $^{19}$; además de la importancia que adquiere el síndrome de ALPS en la evolución de nuestros pacientes, lo que tampoco ha sido descrito en publicaciones nacionales.

La premisa de intentar utilizar antimicrobianos tradicionales ${ }^{23}$ es importante, por la evidente disminución del desarrollo de nuevos antibacterianos ${ }^{41}$. Además, el uso indiscriminado de cefalosporinas de tercera generación ha determinado la descripción de aislados resistentes a ésta y otras familias de antibacterianos, con ejemplos tan conocidos como cepas de bacilos gramnegativos productoras de $\beta$-lactamasas de espectro extendido, cepas hiperproductoras de cefalosporinasas (AmpC), además de Clostridium difficile y Enterococcus sp resistentes a vancomicina ${ }^{42-45}$; determinando, finalmente -para el tratamiento de infecciones producidas por bacilos gramnegativos resistentes- el uso creciente de carbapenémicos ${ }^{46}$. Es importante destacar que la escasa utilización de la información microbiológica en la práctica clínica es una conducta frecuente en nuestro medio como en el extranjero; más aún, es avalada en la literatura médica internacional ${ }^{24,25} \mathrm{y}$ también en Chile ${ }^{26}$. En este contexto, el fundamento más frecuente para el uso masivo de cefalosporinas de tercera generación, especialmente ceftriaxona, en el tratamiento de infecciones extra-meníngeas producidas por cepas de $S$. pneumoniae susceptibles a penicilina, está dada por las propiedades farmacocinéticas de esta molécula, que permiten su administración una vez al día y las consideraciones farmacoeconómicas derivadas $^{47-49}$; más que por literatura, con adecuado nivel de evidencia que demuestre una mayor eficacia clínica de ceftriaxona versus penicilina $\mathrm{G}$.

También debe comentarse que los puntos de corte del CLSI para infección neumocóccica, habitualmente utilizados en el área de enfermedades infecciosas y microbiología clínica, fueron determinados para infección meníngea ${ }^{49,50}$. Lamentablemente, estos puntos de corte se han extrapolado y aplicado en forma sistemática a infecciones fuera del SNC. Sin embargo, del punto de vista farmacocinético y farmacodinámico, es conocido que en la infección respiratoria baja la concentración de penicilina $G$, es mucho más alta que la alcanzada en el espacio subaracnoídeo ${ }^{49-51}$. Así, una serie de publicaciones enfatiza la necesidad de nuevos puntos de corte para infección neumocócica en focos no meníngeos. Destaca, particularmente, el Drugresistant Streptococcus pneumoniae Therapeutic Group que, sobre la base de varios estudios clínicos, sugiere nuevos puntos de corte para infección neumocóccica en el tracto respiratorio inferior; así los aislados con CIM hasta $1 \mu \mathrm{g} / \mathrm{ml}$ deberían ser considerados como susceptibles, intermedios aquéllos con valores de $2 \mu \mathrm{g} / \mathrm{ml}$ y resistentes los aislados con valores de CIM iguales o mayores a $4 \mu \mathrm{g} / \mathrm{ml}^{52-54}$.

El corolario de nuestra línea de pensamiento es que 
cualquier conducta que tienda a utilizar penicilinas en lugar de cefalosporinas permitirá, aunque sea en forma marginal, modular la creciente descripción de resistencia en bacilos gramnegativos $\mathrm{y}$, por ende, ayudar a preservar la utilidad clínica de cefalosporinas y carbapenémicos.

Agradecimientos: A Mario Calvo A, por su opinión sobre el trabajo y asesoría estadística; a Joaquín Bermejo por su opinión, sugerencias y apoyo.

\section{Resumen}

La descripción creciente de Streptococcus pneumoniae resistente a penicilina, ha llevado a la recomendación de emplear cefalosporinas de tercera generación para el tratamiento de las infecciones neumocócicas graves. Como consecuencia, los médicos clínicos no prescriben, usualmente, antimicrobianos de primera línea, a pesar de disponer de estudios de susceptibilidad in vitro. Con el propósito de evaluar el impacto de la asesoría del infectólogo para el uso adecuado de los estudios de susceptibilidad, se compararon dos series: un control histórico (1998-2002, $\mathrm{n}=50)$ y un grupo prospectivo en que sí hubo asesoría infectológica (2003-2006, $\mathrm{n}=60)$. En ambas series, la neumonía fue el foco principal de bacteriemia y alcoholismo/falla hepática los factores asociados más frecuentes. En el primer grupo, 48 aislados resultaron susceptibles a penicilina por prueba de difusión en agar y en uno de 36 pacientes con neumonía, se efectuó cambio en la terapia antimicrobiana a bencilpenicilina. En el grupo prospectivo, hubo 56 cepas susceptibles y las cuatro restantes fueron clasificadas como de susceptibilidad intermedia, de acuerdo con los valores de CIM. Se cambió de antimicrobiano en 36 de 50 pacientes con neumonía neumocóccica bacteriémica $(\mathrm{p}<0,05)$. La evaluación hecha por el infectólogo tuvo un impacto positivo en el uso de información microbiológica con fines terapéuticos.

\section{Referencias}

1.- Edwards K M. Pneumococcal infections: therapeutics strategies and pitfalls. The pneumococcus. Tuomanen EI, Mitchell TJ, Morrison DA, Spratt BG editors. Washington: ASM Press American Society for Microbiology 2004; p 314-30.

2.- Trampuz A, Widmer A F, Fluckiger U, Haenggi M, Frei R, Zimmerli W. Changes in the epidemiology of pneumococcal bacteremia in a Swiss university hospital during a 15 year period, 1986-2000. Mayo Clin Proc 2004; 79: 604-12.

3.- Hook E W, Horton C A, Schaberg D R. Failure of intensive care unit support to influence mortality from pneumococcal bacteremia. JAMA 1983; 249: 1055-7.

4.- Austrian R, Gold J. Pneumococcal bacteremia with special reference to bacteremic pneumococcal pneumonia. Ann Intern Med 1964; 60-759-76.

5.- Fine M J, Smith M A, Carson C A, Mutha S S, Sankey S S, Weissfeld L A, et al. Prognosis and outcomes of patients with community-acquired pneumonia. A metaanalysis. JAMA 1996; 275: 134-41.

6.- Saldías F, O’Brien A, Gederlini A, Farías G, Díaz A. Neumonía adquirida en la comunidad en el anciano inmunocompetente que requiere hospitalización. Cuadro clínico, factores pronósticos y tratamiento. Arch Bronconeumol 2003; 39: 333-40.
7.- Shariatzadeh M R, Huang J Q, Tyrrell G J, Johnson M M, Marrie T J. Bacteremic pneumococcal pneumonia. A prospective study in Edmonton and neighboring municipalities. Medicine 2005; 84: 147-61.

8.- Mufson M A, Stanek R J. Bacteremic pneumococcal pneumonia in one American city: a 20-year longitudinal study, 19781997. Am J Med 1999; 107: 34-43.

9.- Gransden W R, Eykyn S J, Phillips I. Pneumococcal bacteraemia: 325 episodes diagnosed at St Thomas's hospital Br Med J 1985; 290: 505-8.

10.- Ambrose K, Stephens D S. Macrolide, quinolone, and other non $\beta$-lactam antibiotic resistance in Streptococcus pneumoniae. The pneumococcus. Tuomanen EI, Mitchell TJ, Morrison DA, Spratt BG editors. Washington: ASM Press American Society for Microbiology 2004; p 350-66.

11.- Niederman M S, Mandell L A, Anzueto A, Bass J B, Broughton W A, Campbell G D, et al. Guidelines for the management of adults with community-acquired pneumonia. Diagnosis, assessment of severity, antimicrobial therapy, and prevention. Am J Respir Crit Care Med 2001; 163: 1730-54.

12.- Mandell L A, Bartlett J G, Dowell S F, File Jr T M, Musher D M, Whitney C. Update of practice guidelines for the management of community-acquired pneumonia in immunocompetent adults. Clin Infect Dis 2003; 37: 1405-33.
13.- Lagos R, Muñoz A, Valenzuela M T, Heitman I, Levine M M. Population-based surveillance for hospitalized and ambulatory pediatric invasive pneumococcal disease in Santiago, Chile. Pediatric Infect Dis J 2002; 21: $1115-23$.

14.- Inostroza J, Trucco O, Prado V, Vinet A M, Retamal G, Ossa G, et al. Capsular serotype and antibiotic resistance of Streptococcus pneumoniae isolates in two chilean cities. Clin Diagn Lab Immunol 1998; 5: 176-80.

15.- Soler T, Salamanca L, Arbo G, Molina E. Estudio de sensibilidad in vitro de cepas de Streptococcus pneumoniae de infecciones respiratorias bajas en el Instituto Nacional del Tórax (INT). Rev Méd Chile 2002; 130: 304-8.

16.- Fernández M, Zagolín M, Ruiz M, Martínez M A, Díaz J C. Neumonía adquirida en la comunidad que se hospitaliza. Estudio etiológico. Rev Méd Chile 2003; 131: 498-504.

17.- Díaz A, Alvarez M, Callejas C, Rosso R, Schnettler K, Saldías F. Clinical picture and pronostic factors for severe communityacquired pneumonia in adults admitted to the intensive care. Arch Bronconeumol 2005; 41: $20-6$.

18.- Noriega L M, González P, Canals C, Michaud P. Streptococcus pneumoniae septicemia. Análisis of 40 cases. Rev Méd Chile 1994; 122: 1385-92.

19.- Rioseco M L, Riquelme R. Neumonía 
neumocócica bacterémica en 45 adultos inmunocompetentes hospitalizados. Cuadro clínico y factores pronósticos. Rev Méd Chile 2004; 132: 588-94.

20.- Altuzarra R, Valenzuela M T, Trucco O, Inostroza J, Granata P, Fleiderman J, et al. Portación nasal de Streptococcus pneumoniae en adulto mayor y su respuesta frente a la vacunación antineumocócica. Rev Méd Chile 2007; 135: 160-6.

21.- Saldías F, Flores L J, Torres C, García P, Díaz F. Susceptibilidad a antimicrobianos de Streptococcus pneumoniae en población infantil y adulta de Santiago. Período 19972003. Rev Méd Chile 2005; 133: 42-9.

22.- Díaz A, Labarca J, Pérez C, Ruiz M, Wolff M. Tratamiento de la neumonía del adulto adquirida en la comunidad. Rev Chil Infect 2005; 22(Supl 1): 52-66.

23.- Mella S, Blamey R, Riedel G, Aylwin M, Chabouty $H$. Utilidad de penicilina $G$ en el tratamiento de la neumonía neumocócica. Rev Chil Infect 2005; 22: 377-8.

24.- Waterer G W, Jennigs S G, Wunderink R G. The impact of blood cultures on antibiotic therapy in pneumococcal pneumonia. Chest 1999; 116: 1278-81.

25.- Campbell S G, Marrie T J, Anstey R, Dickinson G, Ackroyd-Stolarz S. The contribution of blood cultures to the clinical management of adult patients admitted to the hospital with community-acquired pneumonia. A prospective observational study. Chest 2003; 123: 1142-50.

26.- Rioseco M L, Riquelme R. Replica a la carta bacteriemia neumocócica y antibióticos. Rev Méd Chile 2004; 1260-1

27.- White A C Jr, Atmar R L, Wilson J, Cate T R, Stager C E, Greenberg S B. Effects of requiring prior authorization for selected antimicrobials expenditures, susceptibilities, and clinical outcomes. Clin Infect Dis 1997; 25: 230-9.

28.- Shlaes D M, Gerding D N, John Jr, JF, Craig W A, Bornstein DL, Duncan RA, et al. Society for Healthcare Epidemiology of America and Infectious Diseases Society of America Joint Committee on the Prevention of Antimicrobial Resistance: Guidelines for the prevention of antimicrobial resistance in hospitals. Clin Infect Dis 1997; 25:584-9.

29.- Elliot J, Facklam R. Manual de laboratorio para la identificación y prueba de susceptibilidad a los antimicrobianos de importancia para la Salud Pública en el mundo en desarrollo. Haemophilus infuenzae, Neisseria meningitidis, Streptococcus pneumoniae, Neisseria gonorrhoeae, Salmonella serotipo Typhi, Shigella y Vibrio cholerae. Centros para el Control y la Prevención de Enferme- dades: Centro Nacional para las Enfermedades Infecciosas (CDC) y Organización Mundial de la Salud, Enfermedades Transmisibles: Vigilancia y Respuesta. 2004.

30.- Dowson C G. What is a pneumococcus? In: The pneumococcus. Tuomanen EI, Mitchell TJ, Morrison DA, Spratt BG editors. Washington: ASM Press American Society for Microbiology 2004; p 3-14.

31.- Janoff E N, Rubins J. Immunodeficiency and invasive pneumococcal disease. In: The pneumococcus. Tuomanen EI, Mitchell TJ, Morrison DA, Spratt BG editors. Washington: ASM Press American Society for Microbiology 2004; p 252-80.

32.- Musher D M. Streptococcus pneumoniae. In Mandell GL, Bennett JE, Dolin R, editors. Mandell, Douglas and Bennett's Principles and Practice of Infectious Diseases, Elsevier, $6^{\text {th }}$ Ed 2005; p 2392-411.

33. - Pekka Nuorti J, Butler J C, Farley M M, Harrison L H, McGeer A, Kolczak M S, et al. Cigarette smoking and invasive pneumococcal disease. N Engl J Med 2000; 342: 681-9.

34.- Clinical and Laboratory Standards Institute. Performance standards for antimicrobial disk susceptibility tests. Approved Standards- $9^{\text {th }}$ edition. CLSI document M2-A9. Clinical and Laboratory Standards Institute, Wayne, Pa. 2006 .

35.- Clinical and Laboratory Standards Institute. Performance standards for antimicrobial susceptibility testing; Sixteenth Informational Supplement. CLSI document M100-S16. Clinical and Laboratory Standards Institute. Wayne, Pa. 2006.

36.- Perlino C, Rimland D. Alcoholism, leukopenia and pneumococcal sepsis. Am Rev Res Dis 1985; 132: 757-60.

37. - Solomkin J S, Fant W K, Rivera J O, Alexander J W. Randomized trial of imipenem-cilastatin versus gentamicin and clindamycin in mixed flora infections. Am $\mathrm{J}$ Med 1985; 78 (Suppl 6A): 85-91.

38.- Podnos Y D, Jiménez J C, Wilson S E. Intra-abdominal sepsis in elderly persons. Clin Infect Dis 2002; 35: 62-8.

39.- Valdivieso F, Trucco O, Prado V, Díaz M C, Ojeda A. Antimicrobial resistance of agents causing urinary tract infections in 11 chilean hospitals. PRONARES project. Rev Méd Chile 1999; 127: 1033-40.

40. - Wolff M. Rol actual de los antibióticos tradicionales. Rev Chil Infect 1994; 11: 201-9.

41.- Spellberg B, Powers J, Brass E, Miller L G, Edwards J E. Trends in antimicrobial drug development: implications for the future. Clin Infect Dis 2004; 38: 1279-86.
42.- Dancer S J. The problem with cephalosporins. J Antimicrob Chemother 2001; 48: 463-78.

43.- Medeiros A A. Evolution and dissemination of $\beta$-lactamases accelerated by generations of $\beta$-lactam antibiotics. Clin Infect Dis 1997; 24 (Suppl 1): 19-45.

44.- Hanson N D. AmpC $\beta$-lactamases: what do we need to know for the future? J Antimicrob Chemother 2003; 52: 2-4.

45.- Patterson J E. Extended-spectrum betalactamases. Sem Res Crit Care Med 2003; 24: 79-87.

46.- Chambers H F. Other $\beta$-lactam antibiotics. In Mandell GL, Bennett JE, Dolin R, editors. Mandell, Douglas and Bennett's Principles and Practice of Infectious Diseases, Elsevier, $6^{\text {th }}$ Ed 2005; p 311-8.

47.- Adu A, Armour C L. Drug utilization review (DUR) of the third generation cephalosporins. Drugs 1995; 50: 423-39.

48.- Andes D R, Craig W A. Cephalosporins. In Mandell GL, Bennett JE, Dolin R. Eds. Mandell, Douglas and Bennett's Principles and Practice of Infectious Diseases. Elsevier 2005, $6^{\text {th }}$ Ed, USA 2005; p: 294-311.

49. - Feldman C. Clinical relevance of antimicrobial resistance in the management of pneumococcal community acquired pneumonia. J Lab Clin Med 2004; 143: 269-83.

50.- Musher D M, Bartlett J G, Doern G V. A fresh look at the definition of susceptibility of Streptococcus pneumoniae to betalactam antibiotics. Arch Intern Med 2001; 161: 2538-44.

51.- Hieber J P, Nelson J D. A pharmacologic evaluation of penicillin in children with purulent meningitis. N Engl J Med 1977; 297: 410-13.

52.- Heffelfinger J D, Dowell S F, Jorgensen J H, Klugman K, Mabry L R, Musher D M, et al. Management of community-acquired pneumonia in the era of pneumococcal resistance. A report from the Drug-resistant Streptococcus pneumoniae Therapeutic Working Group. Arch Intern Med 2000; 160: 1399-408.

53. - Pallares R, Liñares J, Vadillo M, Cabellos C, Manresa F, Viladrich P F, et al. Resistance to penicillin and cephalosporin and mortality from severe pneumococcal pneumonia in Barcelona, Spain. N Engl J Med 1995; 333: 474-80.

54.- Feikin D R, Schuchat A, Kolczak M, Barrett N L, Harrison L H, Lefkowitz L. Mortality from invasive pneumococcal pneumonia in the era of antibiotic resistance, 1995-1997. Am J Public Health 2000; 90: 223-9 